\title{
Highly Multiplexed Targeted Proteomics Acquisition on a TIMS-QTOF
}

Antoine Lesur ${ }^{1}$, Pierre-Olivier Schmit ${ }^{2}$, François Bernardin ${ }^{1}$, Elisabeth Letellier ${ }^{4}$, Sven Brehmer ${ }^{3}$, Jens Decker $^{3}$, Gunnar Dittmar ${ }^{*}$

1 Quantitative Biology Unit, Luxembourg Institute of Health, 1a Rue Thomas Edison, L-1445 Strassen, Luxembourg 2 Bruker Daltonics S.A., 34 rue de Industrie, 67166 Wissembourg, France

3 Bruker Daltonik GmbH, Fahrenheitstrasse 4, 28359 Bremen, Germany

4 Department of Life Sciences and Medicine, University of Luxembourg, 6 Avenue du Swing, L-4367 Campus Belval, Luxembourg

* correspondence should be addressed to gunnar.dittmar@lih.lu 
supplementary figure 1:

1 $6 \quad 12 \quad 16$

89

97

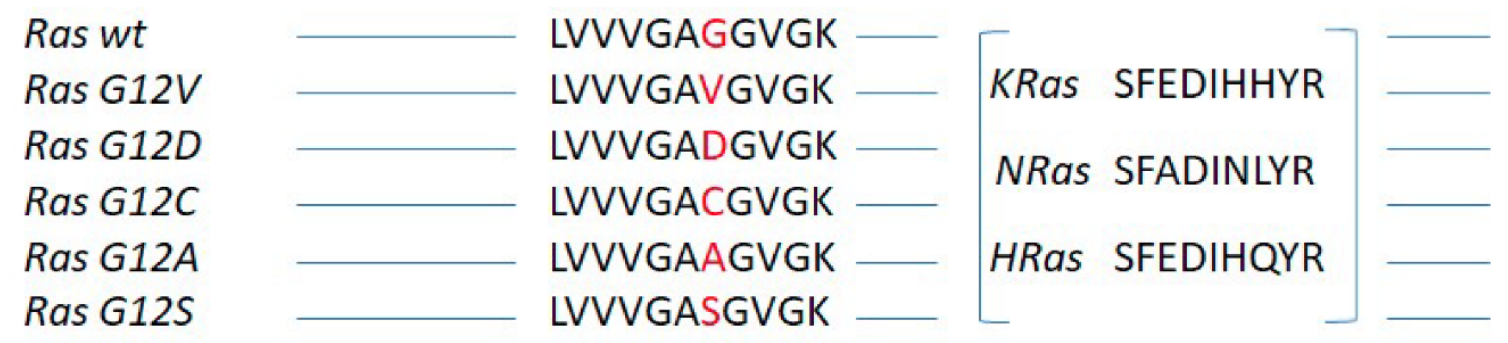

Sequence alignment of the signature peptides employed for the screening of Ras mutation status in the cancer cell lines. 
TIMS disabled

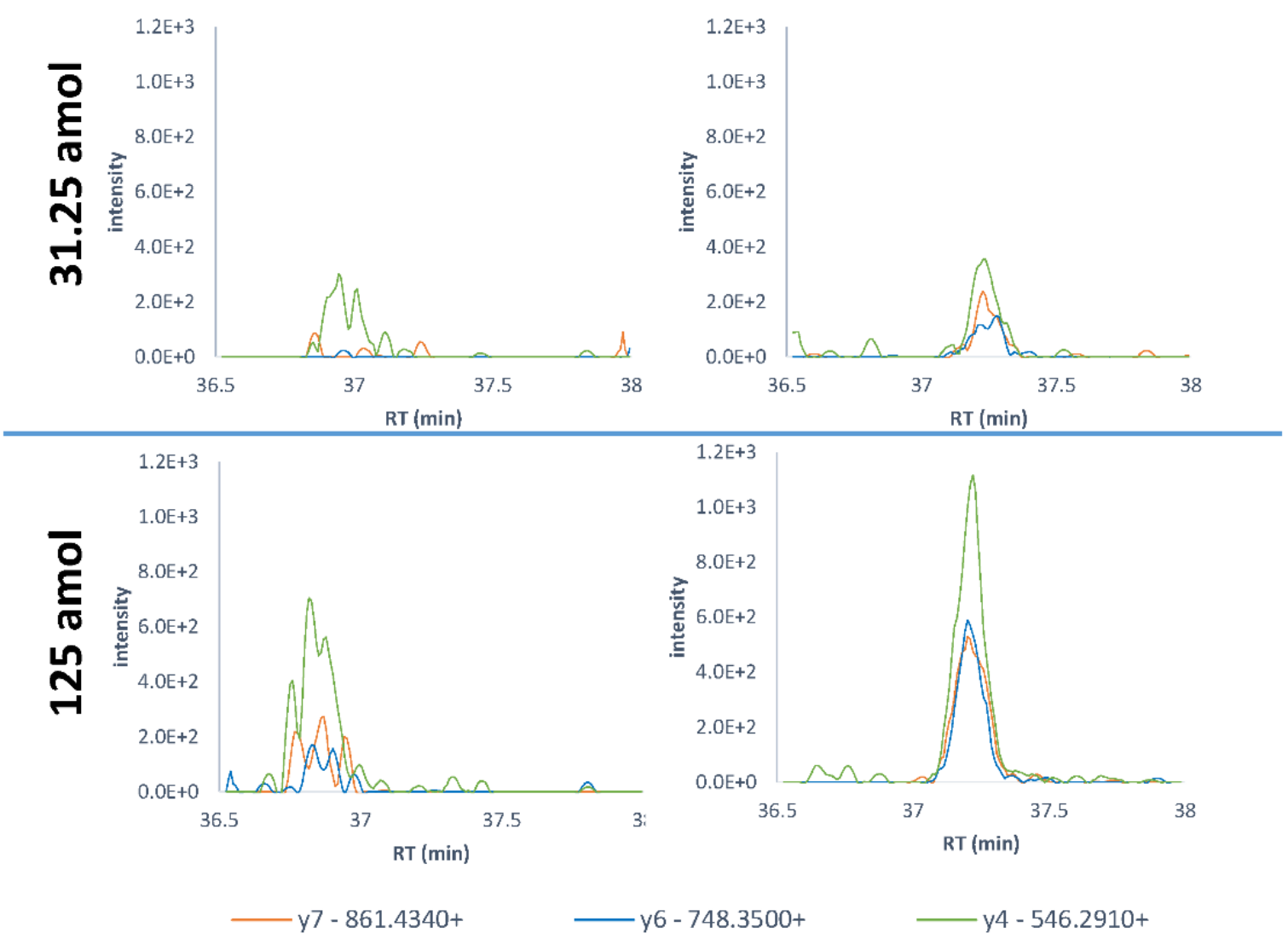

Illustration of the sensitivity improvement in TIMS-PRM mode versus "standard Q-TOF" PRM for the peptide TLLSDPTYR. Precursor ions are physically accumulated for $100 \mathrm{~ms}$ and then eluted in $5 \mathrm{~ms}$ width peaks into the Q-TOF. It improves sensitivity to compare with signal summation in a standard Q-TOF setup. 


\section{Supplementary figure 3:}
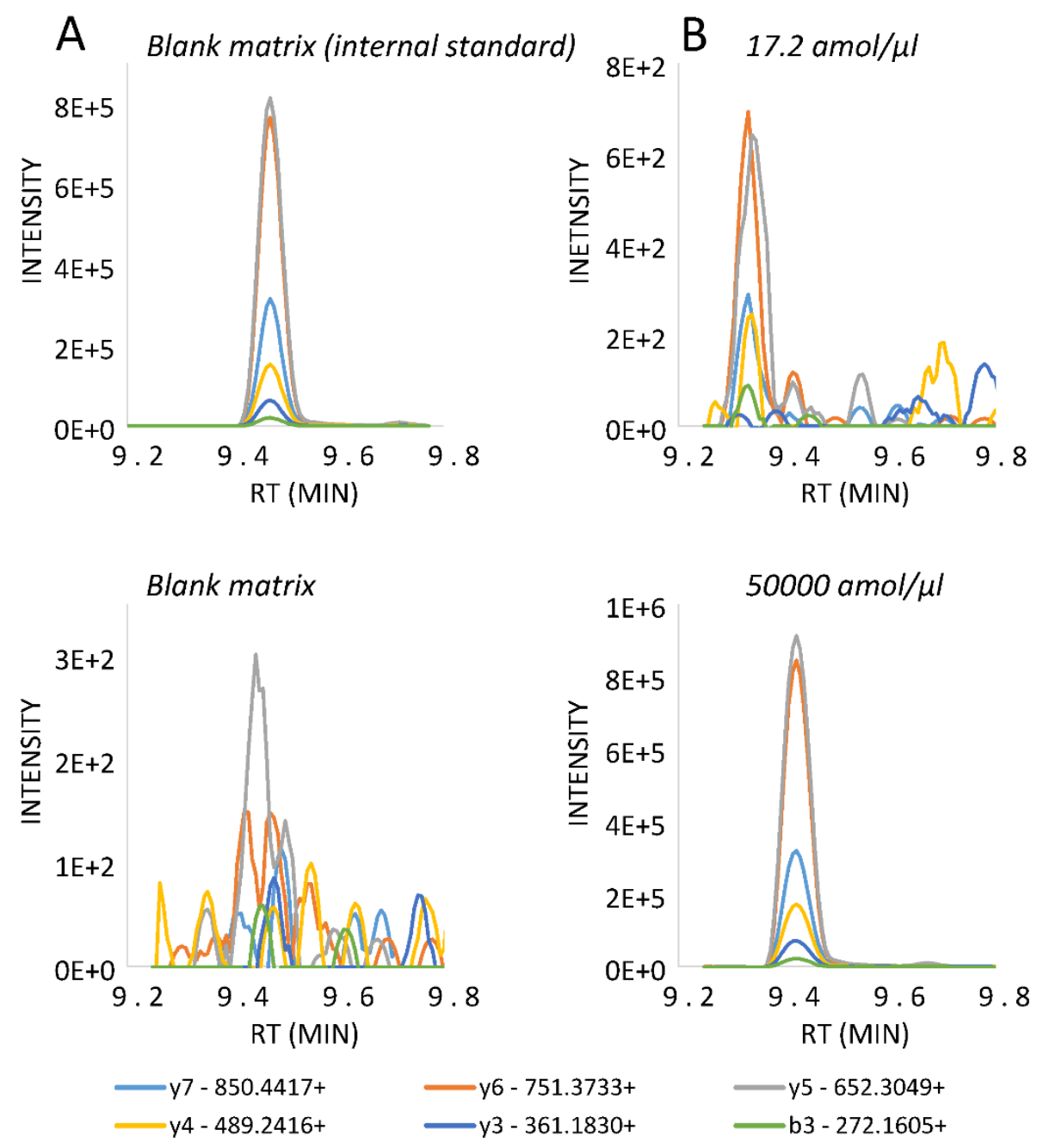

Representative prm-PASEF traces of the peptide ATVVYQGER.

A. prm-PASEF trace of the light labeled internal peptide (upper). Trace of the background signal of the heavy peptide channel on the biological matrix (lower). B. Representative signal of the lowest concentration injection (17.2 amol/ $\mu \mathrm{l}$, upper) and the highest injection of $(50000 \mathrm{amol} / \mu \mathrm{l}$, lower $)$ of the heavy labeled peptide. 


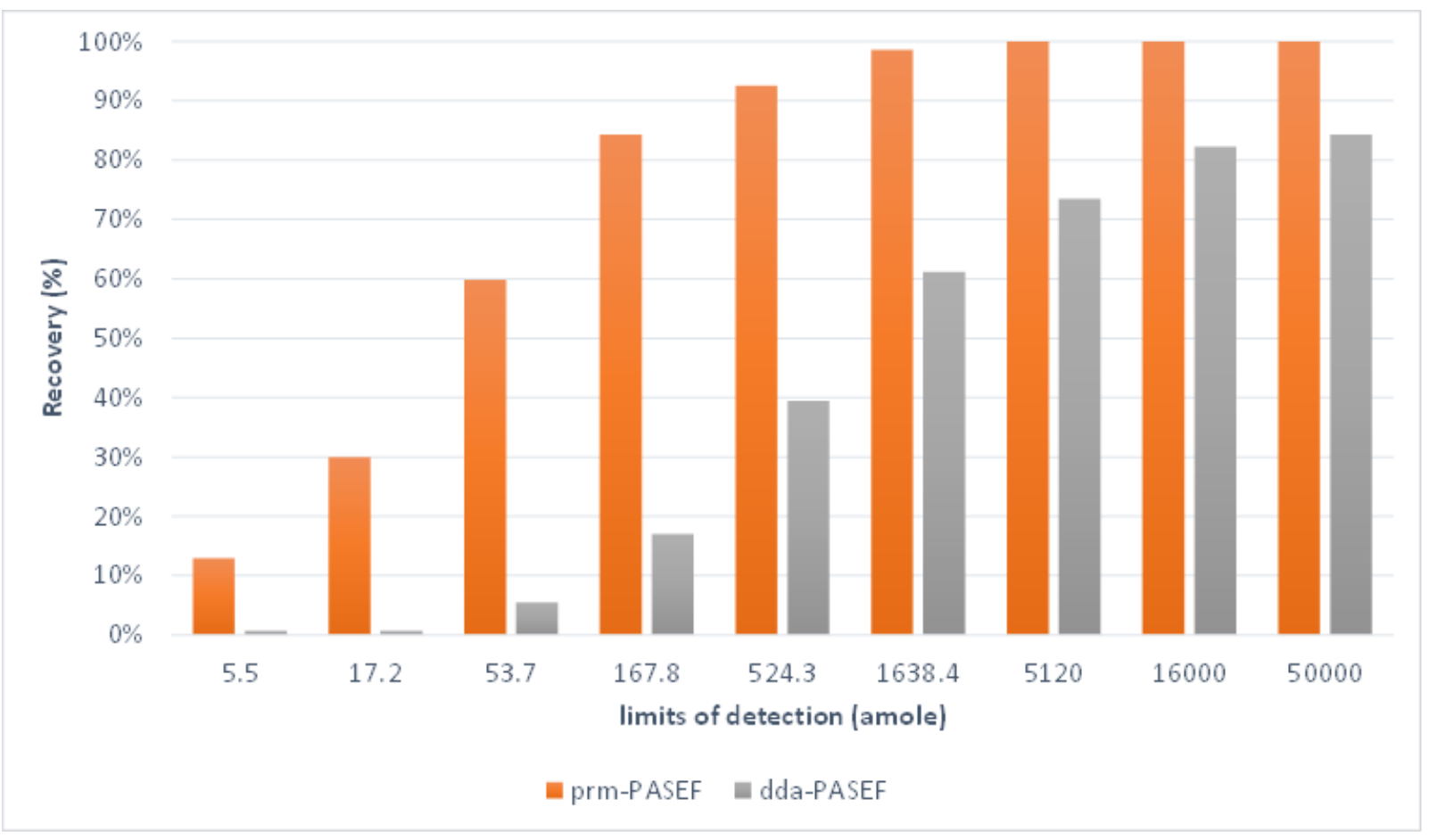

Comparison of detection limit between prm-PASEF and dda-PASEF

Increasing concentrations of $147 \mathrm{AQUA}$ peptides ranging from 5.5 to $50000 \mathrm{amol} / \mu \mathrm{l}$ were spiked in a Hela cell extract samples and analyzed by prm-PASEF and dda-PASEF, using the same MS and chromatography conditions employed in the main manuscript.

For the prm-PASEF experiment, the identification of the AQUA peptides were validated in the prm-PASEF by the calculation of the cosine of the spectral contrast angle ${ }^{1}$ between the XICs of the product ions of the peptides using the concentration $5120 \mathrm{amole} / \mu \mathrm{l}$ as a reference. Data points associated with a cosine of the spectral contrast angle $<0.98$ were eliminated. ${ }^{2}$ The formula is as follows, where $\mathrm{A}$ is the area the product ions XICs with ref the area corresponding to the concentration 5120 amole/ $\mu l$.

$$
\cos (\theta)=\frac{\sum_{i=1}^{n}\left(A_{\exp _{i}} \times A_{\text {ref }_{i}}\right)}{\sqrt{\sum_{i=1}^{n}\left(A_{\text {exp }_{i}}\right)^{2} \times \sqrt{\sum_{i=1}^{n}\left(A_{\text {ref }_{i}}\right)^{2}}}}
$$

For the dda-PASEF experiment, data were analyzed with Mascot 2.6.0 using a Uniprot human proteome database and using the variable modification $\mathrm{K}+8 \mathrm{Da}$ and $\mathrm{R}+10 \mathrm{Da}$.

References:

1. Wan KX, Vidavsky I, Gross ML. Comparing similar spectra: from similarity index to spectral contrast angle. Journal of the American Society for Mass Spectrometry.2002; 13:85-88.

2. Gallien S, Domon B. Detection and quantification of proteins in clinical samples using high resolution mass spectrometry. Methods. 2015; 81:15-23. 